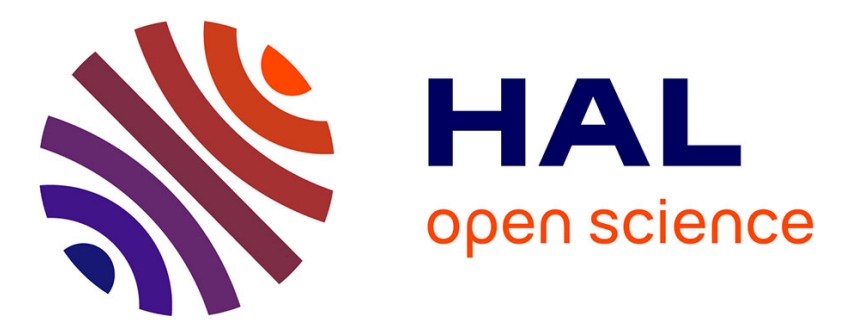

\title{
ARFBF MODEL FOR NON STATIONARY RANDOM FIELDS AND APPLICATION IN HRTEM IMAGES
}

\author{
Zhangyun Tan, Abdourrahmane Atto, Olivier Alata, Maxime Moreaud
}

\section{To cite this version:}

Zhangyun Tan, Abdourrahmane Atto, Olivier Alata, Maxime Moreaud. ARFBF MODEL FOR NON STATIONARY RANDOM FIELDS AND APPLICATION IN HRTEM IMAGES. IEEE International Conference on Image Processing (ICIP) 2015, Sep 2015, Québec City, Canada. hal-01175843

\section{HAL Id: hal-01175843 \\ https://hal.science/hal-01175843}

Submitted on 13 Jul 2015

HAL is a multi-disciplinary open access archive for the deposit and dissemination of scientific research documents, whether they are published or not. The documents may come from teaching and research institutions in France or abroad, or from public or private research centers.
L'archive ouverte pluridisciplinaire HAL, est destinée au dépôt et à la diffusion de documents scientifiques de niveau recherche, publiés ou non, émanant des établissements d'enseignement et de recherche français ou étrangers, des laboratoires publics ou privés. 


\title{
ARFBF MODEL FOR NON STATIONARY RANDOM FIELDS AND APPLICATION IN HRTEM IMAGES
}

\author{
Zhangyun TAN ${ }^{1}$, Abdourrahmane M. ATTO', Olivier ALATA ${ }^{2}$, Maxime MOREAUD $^{3}$ \\ ${ }^{I}$ LISTIC, EA 3703, University Savoy Mont Blanc - France \\ ${ }^{2}$ Lab. Hubert Curien, CNRS UMR 5516 - Jean Monnet University of Saint-Etienne - France \\ ${ }^{3}$ IFP Energies Nouvelles, BP 3, 69360 Solaize - France
}

\begin{abstract}
This paper presents a new model called Autoregressive Fractional Brownian Field (ARFBF) for analyzing textures which contain stationary and non-stationary components. The paper also proposes two estimation methods for the parameter of an isotropic fractional Brownian field based on Wavelet Packet (WP) spectrum: the Log-Regression on Diagonal WP spectrum (Log-RDWP) and the LogRegression on Polar representation of WP spectrum (LogRPWP). The Log-RPWP method provides a better estimation performance for small size images. We show the interest of ARFBF model and Log-RPWP for characterizing High-Resolution Transmission Electron Microscopy (HRTEM) images.
\end{abstract}

Index Terms - Autoregressive Field, Fractional Brownian Field, Texture, Transmission Electron Microscopy

\section{INTRODUCTION}

Texture is one of the most important features for visual perception and an informative component in almost all imaging systems (optical, radar, sonar, ultrasound, X-ray, microscopy, etc.). In this paper, we derive statistical models for HRTEM textures. The HRTEM textures correspond to the observation of material microstructures at nanometer scale. Possible applications of modeling these textures have some environmental impacts:

- $\quad$ refining with low environmental impact using more specialized catalysts,

- $\quad$ improving post-treatment for making standard and hybrid motors cleaner and more efficient by means of a better understanding of soot nanostructure.

Recently, many developments in visualization and analysis of catalysts using TEM micrographs have been proposed [1, 2, 3, 4]. With TEM micrographs at high resolution, (see Figure 1) dark or bright linear patterns (fringes) can be observed and correspond to atomic plane of active phases deposited on the support.

This work was supported by ARC6-TIC, Rhône-Alpes region, France.
The selectivity and activity can be linked to some descriptive parameters of the structure characterization [1] as, for instance, number of fringes per packet, interlayer spacing values and fringe lengths. We propose to use a model-based approach to characterize these fringes.

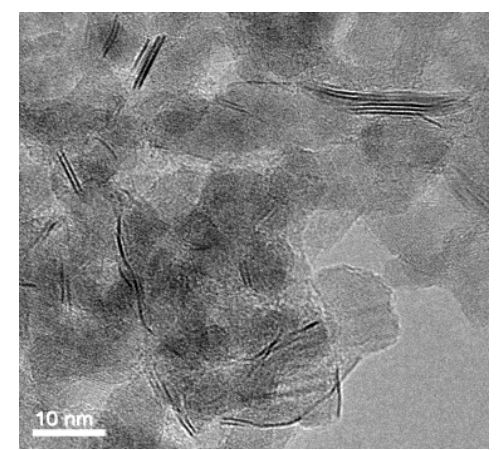

Figure 1: TEM Image of catalyst with active phases (black fringes) deposited on the support.
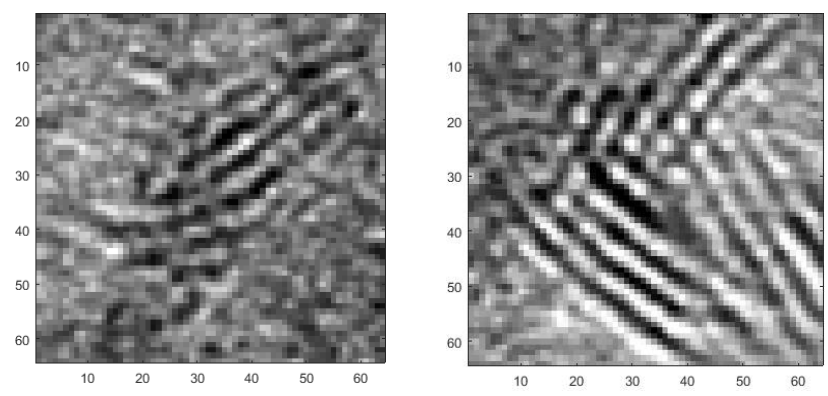

Figure 2: TEM Image close up on active phases of catalyst supports.

The AutoRegressive (AR) model has been studied for many years in order to characterize second order stationary processes. References [5] and [6] show the interest of such a model for textured image segmentation and color texture characterization, respectively.

One classical model for describing many stochastic nonstationary natural phenomena is the fractional Brownian motion (fBm) $[7,8,9,10,11]$, noted as $B_{H}(t) . H$ is called the Hurst parameter. In two dimensions (2D), $\mathrm{fBm}$ is called Fractional Brownian Field (FBF). 
We propose here the AutoRegressive Fractional Brownian Field (ARFBF) model which allows characterizing secondorder stationary properties with its AR part and nonstationary properties with its FBF part.

For estimating the Hurst parameter of ARFBF model, we provide two estimation methods based on Wavelet Packet (WP) spectrum [10]: the Log-Regression on Diagonal WP spectrum (Log-RDWP) and the Log-Regression on Polar representation of WP spectrum (Log-RPWP).

This paper is organized with four sections and we detail them as follows. Section 2 introduces AR and FBF fields. This section also presents the new 2D ARFBF model and its parameter estimation. Section 3 presents the experimental results: estimation performance for Hurst parameter estimation methods and the application of ARFBF to HighResolution Transmission Electron Microscopy (HRTEM) images. Finally section 4 concludes this paper and some perspectives of this work are given.

\section{2D ARFBF MODEL}

\subsection{Autoregressive (AR) and fractional Brownian field (FBF) models}

In this section, we give a brief description about the AR and the FBF models, both in the spatial and the spectral domains.

\subsubsection{Definition and properties of AR model}

Let us define a centered second-order stationary field as $A=\{A(x, y)\},(x, y) \in \mathbb{Z}^{2} . A$ is a $2 \mathrm{D}$ AR process if

$A(x, y)=-\sum_{p_{1}, p_{2} \in D} \beta_{p_{1}, p_{2}} A\left(x-p_{1}, y-p_{2}\right)+E(x, y)(1)$

where $D \subset \mathbb{Z}^{2}$ is a $2 \mathrm{D}$ prediction support. The set $\left\{\beta_{p_{1}, p_{2}}\right\}$ contains the coefficients of the AR process. The spectral density function of an AR process can be written as [6]:

$S_{A R}(u, v)=\frac{\left(\sigma_{e}\right)^{2}}{\left|1+\sum_{p_{1}, p_{2} \in D} \beta_{p_{1}, p_{2}} e^{-i 2 \pi u p_{1}} e^{-i 2 \pi v p_{2}}\right|^{2}}$,

where $\left(\sigma_{e}\right)^{2}$ is the variance of a 2D white Gaussian noise $E=\{E(x, y)\}$.

\subsubsection{Definition and properties of FBF model}

The FBF, noted here as $F=\left\{F_{H}(x, y)\right\}$, is a zero-mean real valued isotropic fractional Brownian field with Hurst parameter $H, 0<H<1$. Its autocorrelation function is

$$
\begin{array}{r}
R_{F}(x, y, s, t)=\frac{\sigma^{2}}{2}\left\{\left(x^{2}+y^{2}\right)^{H}+\left(s^{2}+t^{2}\right)^{H}\right. \\
\left.-\left((x-s)^{2}+(y-t)^{2}\right)^{H}\right\} .
\end{array}
$$

The spectrum of FBF is defined by association and can be written as $[10,12]$ :

$$
S_{F B F}(u, v)=\xi(H) \frac{1}{\left(u^{2}+v^{2}\right)^{H+1}}=\xi(H) \frac{1}{\|f\|^{2 H+2}},
$$

where $\xi(H)=\frac{2^{-(2 H+1)} \pi^{2} \sigma^{2}}{\sin (\pi H) \Gamma^{2}(1+H)},\|f\|=\sqrt{u^{2}+v^{2}}, \sigma^{2}$ is the variance of a white Gaussian noise and $\Gamma$ is the gamma function.

\subsection{Definition and properties of the ARFBF model}

The 2D ARFBF, hereafter denoted $Z=\{Z(x, y)\}$, is defined as the convolution of the AR field $A$ and the FBF $F$.

$$
Z(x, y)=A * F_{H}(x, y)
$$

In the equation (5), the random field $Z$ is an AR when FBF is a white noise $(H=0)$. When $\mathrm{AR}$ is a white noise, then $Z$ behaves as an FBF in the spectral domain. Otherwise, $Z$ is a more general field containing both $\mathrm{AR}$ and FBF contributions. From equation (5), the ARFBF spectrum reduces to:

$$
\begin{aligned}
& S_{A R F B F}(u, v)=S_{A R}(u, v) S_{F B F}(u, v) \\
& =\frac{\left(\sigma_{e}\right)^{2} \xi(H)}{\mid 1+\sum_{p \in D} \beta_{p} e^{-i 2 \pi<f, p>\left.\right|^{2}}} \frac{1}{\|f\|^{2 H+2}} .
\end{aligned}
$$

From equation (6), the spectrum of ARFBF has one singular frequency at the origin (zero frequency point).

Let us denote now one HRTEM image as $I=\{I(x, y)\}$ and its wavelet packet spectrum as $S_{I}$. The image characterization procedure with ARFBF model can be written as follows:

- In the first step, we estimate the parameter $H$ from $S_{I}$ and thus $S_{F B F}$ associated to $I$ is derived. The contribution of the FBF in $I$ is obtained and a residual can be computed.

- In the second step, we remove the contribution of the FBF in $I$, the residual is modeled by an AR model with spectrum defined as follows:

$$
S_{A R}(u, v)=\frac{S_{A R F B F}(u, v)}{S_{F B F}(u, v)},
$$

where $S_{A R F B F}=S_{I}$. From this residual, parameters of the AR part are estimated with classical Yule-Walker method $[5,6]$.

In the next section, we present two estimation methods for Hurst parameter (the first step of the previous proposed procedure).

\subsection{Parameter Estimation}

From equation (4), the spectrum of FBF has the form, 


$$
S_{F B F}(u, v) \sim \frac{1}{\|f\|^{\alpha}} .
$$

This spectrum thus shows an exponential decay and the following provides the Log-RDWP and Log-RPWP estimation methods for the decay parameter $\alpha=2 \mathrm{H}+2$.

In the literature, there exist some methods for estimating the Hurst parameter [12] and [7]. These references present the log-regression parameter estimation based on periodogram for 1D fractional Brownian motion ( $\mathrm{fBm})$. The wavelet packet estimation method has been shown to be more relevant than the periodogram parameter estimation in 1D [10]. Thus we propose, in this paper, two different methods (LogRDWP estimation method and Log-RPWP estimation method) based on $2 \mathrm{D}$ wavelet packet spectrum for the estimation of the Hurst parameter from an image.

\subsubsection{Log-RDWP estimation method}

This estimation method relies on the following formula:

$$
\hat{\alpha}_{\text {Log-RDWP }}=\frac{1}{C} \sum_{0<j<k \leq N} \frac{\log \left(\frac{\hat{S}_{F B F}\left(u_{j}, u_{j}\right)}{\hat{S}_{F B F}\left(u_{k}, u_{k}\right)}\right)}{\log \left(\frac{\left\|f_{k}\right\|}{\left\|f_{j}\right\|}\right)},
$$

where $C=\frac{N !}{2(N-2) !}$ is the number of all possible combinations of the log-ratios, $\hat{S}_{F B F}$ denotes the spectrum estimated from method [10], $\|f\|=\sqrt{u^{2}+v^{2}}, N$ is the number of considered 2D frequencies, $0<j \leq N$ and $j<k \leq N$.

\subsubsection{Log-RPWP estimation method}

The polar estimation method consists in the following steps. In the first step, the spectrum with polar coordinates $W_{I}$ is computed:

$$
W_{I}(r, \theta)=T\left(\widehat{S_{I}}(u, v)\right)
$$

where $\widehat{S_{I}}$ is the spectrum estimated from method [10] of the input image with Cartesian coordinates and $T$ is the Cartesian-to-polar transform. In the second step, averages are done over the angles:

$$
P_{I}\left(r_{i}\right)=\frac{1}{M} \sum_{j=1}^{M} W_{I}\left(r_{i}, \theta_{j}\right) \text { with } 1 \leq i \leq N,
$$

This procedure can be justified by the isotropy of the FBF model considered. In the third step, $\alpha$ is estimated by:

$$
\hat{\alpha}_{\text {Log-RPWP }}=\frac{1}{C} \sum_{0<i<k \leq N} \frac{\log \left(\frac{P_{I}\left(r_{i}\right)}{P_{I}\left(r_{k}\right)}\right)}{\log \left(\frac{r_{k}}{r_{i}}\right)},
$$

where $C=\frac{N !}{2(N-2) !}$ is the number of all possible combinations of the log-ratios, $N$ is the number of averages.

\section{RESULTS AND APPLICATION}

\subsection{Experimental Results}

In order to evaluate the performance of the Log-RDWP and Log-RPWP estimation methods, we generate 10 realizations of a FBF for $\alpha \in\{2.4,2.8,3.2,3.6\}$ and for two different image sizes: $512 \times 512$ and $2048 \times 2048$.

\begin{tabular}{|l|cc|cc|cc|cc|}
\hline Size Image & \multicolumn{7}{|c|}{$512 * 512$} \\
\cline { 2 - 8 } Real Value & 2,4 & \multicolumn{2}{|c|}{2,8} & \multicolumn{2}{|c|}{3,2} & \multicolumn{2}{|c|}{3,6} \\
Estimated & $\alpha$ & $\operatorname{Var}(\alpha)$ & $\alpha$ & $\operatorname{Var}(\alpha)$ & $\alpha$ & $\operatorname{Var}(\alpha)$ & $\alpha$ & $\operatorname{Var}(\alpha)$ \\
\hline RDWP_8 & 1,781 & 0,145 & 2,132 & 0,160 & 2,651 & 0,424 & 3,236 & 0,292 \\
RDWP_12 & 2,186 & 0,038 & 2,553 & 0,086 & 2,993 & 0,053 & 3,553 & 0,090 \\
RDWP_16 & 1,833 & 0,039 & 2,293 & 0,031 & 2,772 & 0,044 & 3,343 & 0,039 \\
RDWP_20 & 2,197 & 0,039 & 2,612 & 0,007 & 3,035 & 0,030 & 3,542 & 0,037 \\
RDWP_24 & 2,145 & 0,045 & 2,688 & 0,019 & 3,050 & 0,019 & 3,510 & 0,018 \\
RDWP_28 & 2,074 & 0,021 & 2,693 & 0,011 & 3,066 & 0,010 & 3,483 & 0,025 \\
RDWP_32 & 1,935 & 0,009 & 2,523 & 0,007 & 2,987 & 0,025 & 3,400 & 0,014 \\
RDWP_64 & 1,778 & 0,008 & 2,487 & 0,006 & 2,936 & 0,006 & 3,433 & 0,002 \\
RPWP_8 & 2,649 & 0,026 & 3,133 & 0,056 & 3,594 & 0,081 & 4,138 & 0,023 \\
RPWP_12 & 2,501 & 0,017 & 2,947 & 0,035 & 3,398 & 0,029 & 3,895 & 0,010 \\
RPWP_16 & 2,486 & 0,004 & 2,948 & 0,016 & 3,435 & 0,018 & 3,942 & 0,003 \\
RPWP_20 & 2,441 & 0,004 & 2,906 & 0,007 & 3,363 & 0,008 & 3,860 & 0,003 \\
RPWP_24 & 2,377 & 0,002 & 2,875 & 0,005 & 3,335 & 0,006 & 3,799 & 0,002 \\
RPWP_28 & 2,322 & 0,002 & 2,844 & 0,003 & 3,312 & 0,005 & 3,767 & 0,002 \\
RPWP_32 & 2,282 & 0,001 & 2,819 & 0,002 & 3,298 & 0,004 & 3,746 & 0,001 \\
RPWP_64 & 1,842 & 0,001 & 2,544 & 0,001 & 3,081 & 0,001 & 3,584 & 0,001 \\
\hline
\end{tabular}

Table 1: Mean values of estimated $\alpha$ and their variances computed from Monte-Carlo simulations on $10 \mathrm{FBF}$ realizations with image size equal to $512 * 512$.

\begin{tabular}{|l|cc|cc|cc|cc|}
\hline Size Image & \multicolumn{7}{|c|}{$2048 * 2048$} \\
\cline { 2 - 8 } Real Value & 2,4 & \multicolumn{2}{|c|}{2,8} & \multicolumn{2}{|c|}{3,2} & \multicolumn{2}{|c|}{3,6} \\
Estimated & $\alpha$ & $\operatorname{Var}(\alpha)$ & $\alpha$ & $\operatorname{Var}(\alpha)$ & $\alpha$ & $\operatorname{Var}(\alpha)$ & $\alpha$ & $\operatorname{Var}(\alpha)$ \\
\hline RDWP_8 & 2,308 & 0,023 & 2,801 & 0,035 & 3,190 & 0,029 & 3,727 & 0,014 \\
RDWP_12 & 2,482 & 0,004 & 2,949 & 0,013 & 3,337 & 0,009 & 3,833 & 0,005 \\
RDWP_16 & 2,241 & 0,004 & 2,748 & 0,008 & 3,193 & 0,007 & 3,642 & 0,001 \\
RDWP_20 & 2,401 & 0,010 & 2,898 & 0,009 & 3,329 & 0,012 & 3,760 & 0,008 \\
RDWP_24 & 2,339 & 0,001 & 2,842 & 0,003 & 3,278 & 0,004 & 3,725 & 0,004 \\
RDWP_28 & 2,322 & 0,002 & 2,834 & 0,002 & 3,282 & 0,002 & 3,733 & 0,002 \\
RDWP_32 & 2,195 & 0,004 & 2,709 & 0,003 & 3,153 & 0,004 & 3,615 & 0,009 \\
RDWP_64 & 1,835 & 0,001 & 2,510 & 0,003 & 3,048 & 0,002 & 3,562 & 0,003 \\
RPWP_8 & 2,688 & 0,010 & 3,209 & 0,009 & 3,701 & 0,015 & 4,260 & 0,007 \\
RPWP_12 & 2,554 & 0,003 & 3,070 & 0,004 & 3,543 & 0,004 & 4,012 & 0,002 \\
RPWP_16 & 2,542 & 0,001 & 3,060 & 0,001 & 3,523 & 0,002 & 3,997 & 0,001 \\
RPWP_20 & 2,498 & 0,001 & 3,011 & 0,001 & 3,462 & 0,001 & 3,940 & 0,001 \\
RPWP_24 & 2,440 & 0,001 & 2,953 & 0,001 & 3,402 & 0,001 & 3,872 & 0,001 \\
RPWP_28 & 2,398 & 0,001 & 2,919 & 0,001 & 3,377 & 0,001 & 3,841 & 0,001 \\
RPWP_32 & 2,346 & 0,001 & 2,892 & 0,001 & 3,357 & 0,001 & 3,819 & 0,001 \\
RPWP_64 & 1,885 & 0,001 & 2,570 & 0,001 & 3,114 & 0,001 & 3,607 & 0,001 \\
\hline
\end{tabular}

Table 2: Mean values of estimated $\alpha$ and their variances computed from Monte-Carlo simulations on $10 \mathrm{FBF}$ realizations with image size equal to $2048 * 2048$. 
RDWP $\mathrm{N}$ and RPWP $\mathrm{N}$ mean that $\alpha$ is estimated by the Log-RDWP method and Log-RPWP estimation method, respectively, where $N$ is the number of samples (see Eq. $9 \&$ 12). We use the Daubechy filter for computing the WP spectrum [10]. The results given in table 1 and table 2 are obtained with a level of decomposition equal to 7 .

By computing the Mean Square Error $\left(M S E=\right.$ Bias $^{2}+$ variance) using values in table 1 and table 2 , we find that, for the small size images, the Log-RPWP method estimates the $\alpha$ parameter better than the Log-RDWP method, particularly when $N$ is equal from 24 to 64 . For the images with a large size, the Log-RDWP method gives comparable results to those of the Log-RPWP method.

\subsection{Application on HRTEM images: characterization of active phases on catalysts supports observed with HRTEM images}

In this section, we present the application of ARFBF model to HRTEM images. The parameters of the model will give a characterization of the observed image.

Spectra of HRTEM images mostly show a significant peak at the origin frequency and an exponential decay in the neighborhood of this peak. This has been emphasized in Figure 3 by providing the periodogram and WP spectrum of an HRTEM image.

The peak at the zero frequency corresponds to slow greylevel variations in the HRTEM image and will be modeled by an FBF. Fundamentally, this peak is not related to the active phase we wish to model. Thus, we propose to remove this peak in order to characterize the main information (active phase).

For removing the FBF part of the image, we estimate alpha from the spectrum $S_{I}$. Then we obtain a spectrum (see equation (7)) associated with the fringes of the image and called $S_{A R}$ (this spectrum characterizes the AR part of the image).

Figure 4 shows the residual image after removing the FBF derived from the input HRTEM image and its spectral representations. The periodogram of figure 4 shows that the energy around the zero frequency has been almost totally removed. We finally estimate the parameter of the AR model from the residual image (see Section 2.2). These parameters allow computing the spectrum given on the right figure of the figure 4 . This spectrum contains a main bump whose shape is associated to the active phase inside the initial HRTEM image. This bump deserves to be further analyzed with morphological tools in order to characterize this active phase.
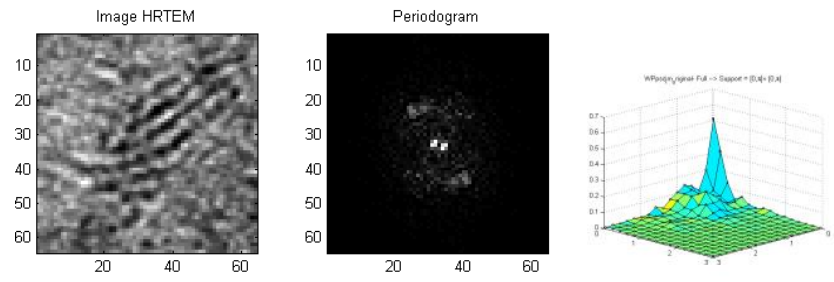

Figure 3: The initial TEM image, its periodogram representing $[-\pi, \pi] \times$ $[-\pi, \pi]$ frequency domain and WP spectrum.
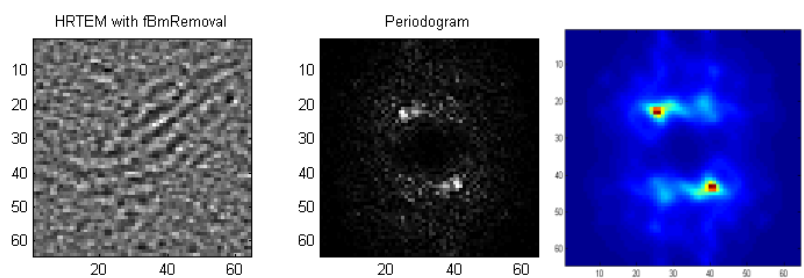

Figure 4: The residual part after removing the FBF contribution of the TEM image, its periodogram representing $[-\pi, \pi] \times[-\pi, \pi]$ frequency domain and power spectral density $([-\pi, \pi] \times[-\pi, \pi]$ frequency domain) computed using the AR parameters estimated from the left image.

\section{CONCLUSION}

In this paper, we propose the ARFBF model in order to characterize both second-order stationary properties and non-stationary properties of textures. We also propose two estimation methods for the parameter of FBF: the LogRDWP and the Log-RPWP. For images with a small size, the Log-RPWP estimation is more relevant than the LogRDWP estimation. We finally apply this model to the characterization of HRTEM images.

In this paper, we have considered one spectral peak for the non-stationary part. In future work, we will extend the ARFBF model to the generalized isotropic fractional fields that can admit several spectral peaks [11]. With such a model, we can address the characterization of an HRTEM image presenting several periodicities with different orientations.

\section{REFERENCES}

[1] M. Moreaud, D. Jeulin, A. Thorel and J. Y. Chane-Ching, "A quantitative morphological analysis of nanostructured ceria-silica composite catalysts", Journal of Microscopy, Vol. 232, Pt 2, pp. 293-305, 2008

[2] M. Moreaud, D. Jeulin, V. Morard and R. Reval, "TEM image analysis and modeling: application to boehmites nanoparticles", Journal of Microscopy, Vol. 245, Pt 2, pp. 186-199, 2012

[3] L. Sorbier, A.S. Gay, A. Fécant, M. Moreaud and N. Brodusch, "Measurement of palladium crust thickness on catalysts by optical microscopy and image analysis", Microscopy and Microanalysis, 2013 
[4] K. Yehliu, R.L. Vander Wal and A.L. Boehman, "A comparison of soot nanostructure obtained using two high resolution transmission electron microscopy image analysis algorithms", Carbon (in press), 2011

[5] O. Alata and C. Ramananjarasoa, "Unsupervised Textured Image Segmentation using 2-D Quarter Plane Autoregressive Support with Four Prediction Support", Pattern Recognition Letters, volume 26, pp. 1069-1081, 2005

[6] I.-U.-H. Qazi, O. Alata, J.-C. Burie, A. Moussa, C. FernandezMaloigne, "Choice of a pertinent color space for color texture characterization using parametric spectral analysis". Pattern Recognition, vol. 44, n 1, pp. 16-31, 2011

[7] G. Rilling, P. Flandrin and P. Gonçalvès, "Empirical Mode Decomposition, Fractional Gauss Noise and Hurst Exponent Estimation", IEEE, 2005

[8] P. Flandrin, "On the spectrum of fractional Brownian Motions", IEEE Transaction on Information Theory, Vol. 35, No. 1, Jan. 1989

[9] A. Ayache, S. Cohen and J. Vehel, "The covariance structure of multi-fractional Brownian motion with application to long dependence", IEEE, ICASSP, 2000

[10] A.M. Atto, D. Pastor and G. Mercier, "Wavelet Packets of fractional Brownian motion: Asymptotic Analysis and Spectrum Estimation", IEEE Transactions on Information Theory, vol. 56, no.9, pp.4741 - 4753, Sept. 2010

[11] A. M. Atto, Z. Tan, O. Alata and M. Moreaud, "NonStationary Texture Synthesis from Random Field Modeling", IEEE ICIP Conference, Paris, France, Oct. 2014

[12] B. Pesquet-Popescu and J. L. Véhel, "Stochastic fractal models for image processing", IEEE Signal Processing Magazine, vol. 19 , no.5, pp.48-62, 2002 